\title{
IdeaHound: Self-sustainable Idea Generation in Creative Online Communities
}

Pao Siangliulue

SEAS Harvard Univ.

Cambridge, MA 02138

paopow@seas.harvard.edu

Joel Chan

$\mathrm{HCIl}, \mathrm{CMU}$

Pittsburgh, PA 15213

joelchuc@cs.cmu.edu

Bernd Huber

SEAS Harvard Univ.

Cambridge, MA 02138

bhb@seas.harvard.edu

\author{
Steven P. Dow \\ $\mathrm{HCII}, \mathrm{CMU}$ \\ Pittsburgh, PA 15213 \\ spdow@cs.cmu.edu
}

Krzysztof Z. Gajos

SEAS Harvard Univ.

Cambridge, MA 02138

kgajos@seas.harvard.edu

Permission to make digital or hard copies of part or all of this work for personal or classroom use is granted without fee provided that copies are not made or distributed for profit or commercial advantage and that copies bear this notice and the full citation on the first page. Copyrights for third-party components of this work must be honored. For all other uses, contact the Owner/Author.

Copyright is held by the owner/author(s).

CSCW' 16 Companion, February 27 - March 02, 2016, San Francisco, CA, USA ACM 978-1-4503-3950-6/16/02.

http://dx.doi.org/10.1145/2818052.2874335

\begin{abstract}
One main challenge in large creative online communities is helping their members find inspirational ideas from a large pool of ideas. A high-level approach to address this challenge is to create a synthesis of emerging solution space that can be used to provide participants with creative and diverse inspirational ideas of others. Existing approaches to generate the synthesis of solution space either require community members to engage in tasks that detract from the main activity of generating ideas or depend on external crowd workers to help organize the ideas. We built IDEAHOUND a collaborative idea generation system that demonstrates an alternative "organic" human computation approach, where community members (rather than external crowds) contribute feedback about ideas as a byproduct of an activity that naturally integrates into the ideation process. This feedback in turn helps the community identify diverse inspirational ideas that can prompt community members to generate more high-quality and diverse ideas.
\end{abstract}

\section{Author Keywords}

creativity support tool; collaborative idea generation

\section{ACM Classification Keywords}

H.5.m [Information interfaces and presentation (e.g., $\mathrm{HCl}$ )]

Miscellaneous 


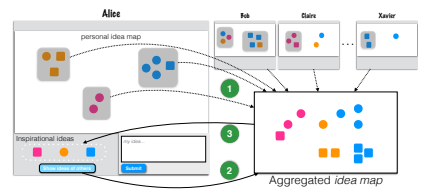

Figure 1: Data collection flow in IDEAHOUND: 1) IDEAHOUND collects information about semantic relationship between ideas and quality of ideas from all users and use them to synthesize a global idea map with semantic relationships betweens all ideas. 2) A user can request to see a set of ideas by others for inspiration. 3) IDEAHOUND consults the idea map and selects a set of inspirational ideas for the user.

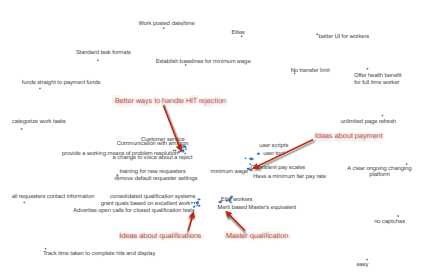

Figure 2: An example of an idea map generated by IDEAHOUND, showing clusters of ideas around different topics.

\section{Introduction}

Large creative online communities provide their members an opportunity to generate and share ideas to solve problems or create something that they care about. Current communities span a wide range of domains from social challenges (OpenIDEO), graphic design (Dribbble) to civic engagement (Cambridge Participatory Budgeting), and have attracted a large number of users who contribute ideas or designs. The members benefit from browsing others' ideas in search of inspiration. The mix of perspectives and expertise among the community members allows creative solutions to emerge in a way unimaginable in the loneinnovator or small-group settings.

However, new challenges arise with this idea generation paradigm. One main challenge facing creative online community members is finding inspirational ideas from a large pool of ideas. While the community gathers a lot of ideas, not all of them are of high quality and many are variations on a small number of popular themes. This is important because prior work has suggested that the ideas people encounter affect their creative output $[4,5]$. To encourage community members to generate creative and diverse ideas, we should provide them with a set of creative and diverse inspirational ideas [5]

The community can identify high-quality, creative ideas using existing social feedback mechanisms (thumb up, stars etc). There are scalable approaches to help the community identify a diverse set of ideas using considerable amounts of human judgement as input $[3,2,1,5]$. These approaches require community members to perform specific tedious, time-consuming activities (tagging, judgements of relative similarity) that do not directly contribute to the ideation process. While some of these activities could be performed by external crowds hired specifically for the purpose, for creative tasks where specialized domain knowledge is required, outsourcing is not a viable option.

Instead of forcing users to do tedious human computation tasks or relying on external crowds, we propose a selfsustainable "organic" human computation approach, in which community members themselves contribute information about semantic relationships among the ideas as a byproduct of an activity that productively integrates with their own ideation process.

In this paper, we present IDEAHOUND, a collaborative idea generation system that embodies the organic approach. The key design principle of IDEAHOUND is to integrate user interactions that 1) provide sufficient information to help the community identify inspirational ideas, and 2) do not detract from users' main task (generating ideas).

\section{System design}

The organic interactions in IDEAHOUND is developed from our observation of how people generate ideas. We observed that people tend to organize existing ideas semantically and evaluatively while generating new ones. One pattern that emerges frequently is spatially arranging existing ideas to see relationship between ideas, detect emerging patterns, and combine two or more concepts to come up with new ones [6]. IDEAHOUND leverages users' natural action of organizing ideas spatially which can also be intrinsically helpful for the users' idea generation.

Figure 1 illustrates the key conceptual design elements of IDEAHOUND. The system provides users with a whiteboard area (see Figure 3) where they can spatially arrange ideas and discretely group ideas together. The system leverages the information conveyed through these individual spatial arrangements to create an aggregate abstract idea map [5] (see Figure 2) that reflects semantic relationship between 
ideas. It collects informations about the quality of ideas through users' normal activities such as removing the ideas from the whiteboard and liking the ideas.

When a user requests to see examples of ideas generated by others, the system samples a set of ideas from the idea map that are predicted to be substantially different from each other and are of high quality. To reduce redundant effort of multiple members exploring the same ideas and ensure that most parts of the solution space are explored, IDEAHOUND also shows ideas sampled from different parts of the idea map to different users.

At the end of each idea generation session, IDEAHOUND provides users a summary visualization of their collective effort based on the generated idea map. The community can use this summary to guide their decision on which ideas to pursue.

\section{Ongoing work}

Our preliminary test of IDEAHOUND with 6 groups of Amazon Mechanical Turk workers $(n=55)$ shows that people engage in organizing ideas as expected and IDEAHOUND generates an idea map that reflects human similarity judgement as well as an idea map generated by micro-task approach in [5]. The comparisons of number of generated ideas and survey responses between the groups that used IDEAHOUND to generate ideas and the groups that use a baseline interface with no organic interactions show that participants in both sets of groups were equally productive and found the system to be equally useful. These results suggest that our organic interactions do not detract users from generating ideas.

We are currently exploring different interfaces that summarize the outcome of idea generation process to help the community understand the problems and make decisions

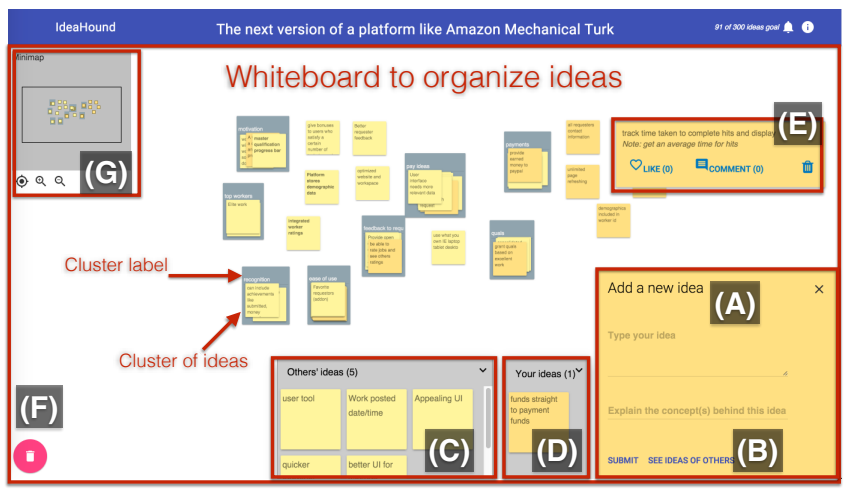

Figure 3: IDEAHOUND interface. (A) A box where users can type and submit their ideas; (B) The request button to see ideas of others, (C) The requested ideas of others arrive, they appear on the Others' ideas Pane; (D) When users submit an idea, it first appears on the Your ideas Pane; Users can move ideas from (C) and (D) to organize on the whiteboard area. When they place an idea close to each other, a cluster will form automatically. $(E)$

When they hover the cursor over an idea, it shows the full text that describes the idea, and show a control panel where users can like the idea, see comments of the ideas, add a comment to the ideas or remove the idea from the workspace. $(F)$ The trashcan. Users can click here to see the ideas that they have removed from the workspace. They can choose to move them back into the workspace. (G) A minimap of the workspace. Users can pan and zoom the whiteboard or control the zoom from the minimap view. 
on ideas to pursue. We are also exploring the effects of different system interventions (e.g., showing ideas of others) on users' creative activities when they are in different cognitive states. In particular, we are developing an integrative application on context-aware devices such as mobile phones where the system can nudge users based on their context such as location and activity.

\section{References}

[1] Paul André, Aniket Kittur, and Steven P. Dow. 2014. Crowd Synthesis: Extracting Categories and Clusters from Complex Data. In Proceedings of CSCW'14 (CSCW '14). ACM, New York, NY, USA, 989-998.

[2] Lydia B Chilton, Greg Little, Darren Edge, Daniel S Weld, and James A Landay. 2013. Cascade: Crowdsourcing taxonomy creation. In Proceedings of the 2013 ACM annual conference on Human factors in computing systems. ACM, 1999-2008.
[3] Richard L Marsh, Joshua D Landau, and Jason L Hicks. 1996. How examples may (and may not) constrain creativity. Memory \& Cognition 24, 5 (1996), 669-680.

[4] Bernard A Nijstad, Wolfgang Stroebe, and Hein FM Lodewijkx. 2002. Cognitive stimulation and interference in groups: Exposure effects in an idea generation task. Journal of experimental social psychology 38, 6 (2002), 535-544.

[5] Pao Siangliulue, Kenneth C. Arnold, Krzysztof Z. Gajos, and Steven P. Dow. 2015. Toward Collaborative Ideation at Scale-Leveraging Ideas from Others to Generate More Creative and Diverse Ideas. In Proceedings of CSCW'15.

[6] Gerald F Smith. 1998. Idea-generation techniques: a formulary of active ingredients. The Journal of Creative Behavior 32, 2 (1998), 107-134. 